\title{
Information and Internet change society: emerging constitution of the social systems
}

\author{
A. Rosický \& A. Pavlíček \\ Department of Systems Analysis, University of Economics, Prague
}

\begin{abstract}
Using adjectives "Information" or "Knowledge" in front of the word society seems to have become popular recently. However, there is an ambiguity with regards to the concepts of information and knowledge and there are problems involved in re-defining them.

Information and communication technologies actually handle only data (or binary-coded symbols), while the meaning of information is created by the recipient according to his own individual knowledge and relative context. The growing volume of shared/mediated "conceptual" information (data) brings us another - often underestimated - problem related to the possibility of different interpretations and thus a danger of fluctuation and turbulence in society. On the other hand, some information systems and/or organizations designed to work with information and knowledge (educational systems or press agencies) unify the perception of common problems and their solutions. The Internet brings us the promising possibility of heterogeneity - but inherently includes the problem of the credibility and validity of shared information.
\end{abstract}

Keywords: cybernetics, conceptual information, cognition, fluctuation, globalisation, information, knowledge, social system, system theory.

\section{Mediated information in global world}

While nobody could doubt that the character of our contemporary world has been fundamentally changed few people can actually understand it. The principal changes in the society seem to be expressed with new terms - coined by usage of prefix POST. There is POSTindustrial society (as opposed to industrial one), there is POSTmodern society, while some years ago there was only modern society. 
- The significance and importance of information brought us term "information society", sometimes replaced by "knowledge society".

- Terms like "globalisation" or "global world" exceed social dimension (society) and describe the growing complexity and perplexity of our planet.

- The term technology is nowadays linked mainly to the information and communication technology, thus causing the attribute "digital" to be used so often. We now talk about the "digital" economy, "digital interface" ...

- Although the principles of cybernetics are not entirely known, some of above mentioned facts are connected with cybernetics. Prefix "CYBER" - originally used in Sci-Fi literature seems to get a whole new meaning now (CYBERculture, CYBERspace and similarly also CYBorg).

It is not aim of this article to analyse the differences and relations between these terms. However, it is obvious that the unifying elements of all the previously mentioned terms are information and fast development of IT and ICT. But information technology is not just the computers and other things related to them. Actually, information technology goes with humankind since the dawn of our civilisation. Of course, historically, information technology was about using different "hardware" tools and skills to preserve and transfer messages based on human language. Milestones of the development were: invention of a scripture, book-print, telegraph. Later came telephone, tape-recorder, TV, the latest addition is technology based on binary coding (which includes all the previous terms). Binary code has made it possible to automatize some processes, to reduce them to the simple operations with binary symbols. The problem is, that such automatization does not take into account the meaning of the recorded information (another example of the same mistake is taking binary digits for figures or even numbers). However, this process of automatization, supported by computer technique (CPU, RAM) and unified syntax made it possible to interconnect computers (and other CPU based systems) into networks.

The Internet, as a world-wide network, has become the most important of these networks. It is phenomenon that radically changes the society by bringing us the possibility to share data (not meaningful information). This phenomenon, together with other media, brings into life the McLuhan's metaphor of "Global Village" [11]. Of course, there is more to the globalization than just informationsharing, but informatization is its fundamental aspect.

The term "informatization" - usually associated with the ICT implementing has arisen by joining together two words - information and automatization; and describes the manipulation with mere symbols, not their meanings. This separates the Internet from the other mass media (institutions), whose main purpose is to communicate information (as a meaning). These mass media (other than the Internet) use advanced technology as well, however its purpose seems to be different. The mass media have an editor's office, staff and redaction - social systems designed to deal with the information. In comparison to them, the Internet is very simple and anonymous - but has three important features- it is (1) on-line, (2) interactive (works two ways) and (3) based on network infrastructure. Traditional media actually seem to use "new media" in some way 
(usually to receive a feedback - SMS messages, email, interactive features of digital TV), however there is still a difference.

Information provided by "traditional" mass-media is "mediated" information - it has been mediated by a middleman. The fact that this middleman actually shifts (intentionally or not) the meaning of the information is often not considered. It brings up the questions of objectivity, purpose of mediation and origin of the information. It demonstrates that in order to fully understand the information, we need to know the context of information [21]. Related issues are: the importance of information in system, especially in social systems (society, economy, company), and the impact and consequences of implementing IS/ICT.

\section{System theory, cybernetics and cognition}

We will try to explain these issues from the points of view of both traditional disciplines, which laid down the groundwork of the process now called informatization. The first issue is cybernetics as it was the first science to recognize the importance of information within a society. Within the limits of traditional thinking of 1950's it formulated quantitative approaches and introduced the "amount of information" that reduces entropy. However, contemporary cybernetics - called 2 nd order cybrentics (oriented on "observing systems") rather pays attention to human knowledge. System theory emphasising complexity and self-organization also frames the area of new thinking (e.g. about Internet). From this perspective is relevant dynamic conception of world with special attention paid to interaction and sharing of conceptual information and/or knowledge.

Systemic concept of the world is generally accepted in the science as well as in real life. Yet, contemporary theories are different to original ones and rigorous systemic thinking confronts us with series of troubles, related to the cognition and understanding the world. Then we also take into consideration the properties of the whole system and its interaction with the other systems within the frame of something called supra-system. Thus, we should focus on following two observations:

- The circular relationship between the part and the whole - it means that every part of the system participates on the whole and vice versa.

- Dynamic character of the processes, in which the patterns of the structure and/or behaviour are formed. Actual structures and behaviour differs from original patterns, they fluctuate round.

That explains the evolution (exceeding the framework of Darwin theory) and explains the material essence of the cognitive processes. Interaction between human (as a physical / biological system) and the environment is described as "learning from the experience" which forms human knowledge [10]. Its human cognition that defines two different world/system concepts: It is real world/system as a physical object and on the other hand it is our idea of it as a model, based on our knowledge. Correspondingly, Hayek [7] describes two different types of order - cosmos as a natural spontaneous order and taxis - order 
embedded /enforced/ by human, according to his own interests and knowledge. Human cognition is based on information exceeding (but including) the traditional concept of information as just a message. As theoretical as it may seems, it lays the ground for understanding the essence and importance of information in the system, including IS/ICT. In this sense, information is a basic category of contemporary science (biology, physics) and in relation to the systemic science and cybernetics is explained by "Unified Theory of Information" [8]. In this concept, information is (together with matter and energy) basic attribute of the real world - defining its versatility, diversity and behaviour. Bateson [1] defines information as: "difference which makes a difference" - in other words: It's not just matter, what defines the properties and character of the system, but also its internal physical organisation ... which we call "information within the system" [Rosický 15].

We can explain the idea by the following examples: Let's take oxygen molecule. Basic physical system. Usually created by 2 oxygen atoms. It is stable and forms a pattern called $\mathrm{O}_{2}$. it is only one of the variety of possible options in real (physical) environment. Under different circumstances, three atom of the same gas can produce a molecule of ozone - $\mathrm{O}_{3}-$ with completely different physical properties.

At the level of living systems emerges new type of information "acquired from the outer system" - the living system consists of parts, which are able to analyse (more or less) the surrounding environment (temperature, illumination, etc.) and react accordingly. Together with increasing complexity of nerve system increase also the elaborateness of the relationships between "inner status" (emotions) and signals (information) from outer system. The information is thus linked to the consciousness/awareness and becomes essential for the processes of learning and (the dynamic nature of) knowledge. This can be described as an information, which is determined by outer and inner system at once - "information applied to system" [Rosický 15]. Such information is of implicit nature, and become "nucleus" of the phenomena like "knowledge", "consciousness" or "intentionality" [16].

These concepts are usually related only to the human beings (anthropological conception), but this is a great mistake/misunderstanding. Systemic science, much like modern cybernetics, points out that such paradigm is unacceptable. For example, some philosophy schools since 1960's have been fighting against the prejudice that human consciousness is somehow exceptional. This approach is advocated also by some exceptional theoreticians of knowledge management [13].

\section{Meaning of conceptual information and knowledge}

Natural language cannot be restricted to just "set of used symbols/signs". The most important part of natural language is syntax (principles, rather than strict rules) for creating the higher structures - messages or statements. Their misinterpretation for /confusion with/ the information itself comes directly from (well known, but persisting) misunderstanding of Shannon's "Information 
theory". The key question, pointed out by Shannon and Weaver [18] is its purposeful ignorance of the meaning of information. The information theory deals rather with the form, than with the content and/or meaning. The relation between information and its meaning is well explained for instance by British Standard BS 3527, Part 1, year 1976, where data and information are defined as:

Information: The meaning that human assigns to data by means of conventions used in their presentation. Data: Representation of facts, concepts, or instruction in a formalized manner suitable for communication, interpretation, or processing by humans or by automatic means.

Well, right now we would like to remind that one could gain information from the environment by all different means, not only by using the language. The meaning of gained information emerges in the process of interpretation, where the central role is played by the individual knowledge/cognition the recipient already have. Also the concept of knowledge is - even between specialists fuzzy and ambiguous (from "the object, you can handle and trade" to the "the state of mind", or "expertise - the way of solving problem". Biological (material) nature of knowledge is explained by Maturana and Varela [10], using the term "knowing" to describe its dynamical and changing nature instead of (static) "knowledge". Biological nature of constitution of knowing has one serious consequence: it relates the knowing only to the one particular organism (body) capable of physical and mental activity. The same (dynamic and individual) concept of knowledge is advocated by representatives of knowledge management (von Krogh et al. [9]). It may sound as a paradox (considering the discipline name), but they express "strong conviction that knowledge cannot be managed, only enabled..." Instead, the continually popular, but underestimated term "tacit knowledge", which is result of self-organizing of autonomous nerve system. It actually correspondents pretty much to the above mentioned "implicit essence of knowledge" and/or "information applied to system".

When knowledge is to be transferred (which is actually essential for human and social systems), it thus must be externalised (expressed) in some way or another. At that moment, the symbols (and signs) of natural language are used. These symbols are about to carry the knowledge from a sender to a recipient. The words, symbols or messages (in natural language) are just the medium to transfer the information. Its successful understanding depends on whether the sender and the recipient share the same intellectual concepts (idea constructs) and same symbol understanding. That's why such information is sometimes called conceptual information or semantic or else human information. In similar sense Tom Stonier put it like this [19, p. 17]:

In contrast to physical information, there exists human information which includes information created, interpreted, organized or transmitted by human beings. The term includes 'data' on the one hand and "knowledge", "insight" and "wisdom" on the other. A datum is a small chunk of information. Usually the term information is thought of as organized data or "facts" organized into a coherent pattern.

In the framework of systemic theory (the theory this article is actually based on) we can explain Stonier's word "organised" as a self-organisation of nerve system. 
Stonier and many others also assume that there is at least conceptual information, which enables to share individual knowledge between each other.

This is the keystone of the human brain, human societies; the key factor of success: the ability to mediate and share human knowledge and experience. That is why knowledge, after all, is not all subjective (as a result of interaction between organism and environment) but rather individual [22]. Knowledge is unique for each and every human being, but at the same time it was shaped by social pressures as well. That is why we can actually understand each other, because we share the social pressures that made us compatible. These "pressures" could be various - but they usually have to do something with the power/authority. It's question of education, question of general culture, question of what (mass) media declare for standards, it is also the question of information technology (including the Internet).

\section{Selected aspects of information and knowledge}

There are usually some deep and complex consequences to any statement, which is just briefly mentioned. But such consequences are usually overlooked or skipped-over silently, unless we take the effort to focus on them. But before we do so in our case, we would like to repeat the previously mentioned meaning of (conceptual) information:

Conceptual information initiates physical activities of individuals as well as whole human communities or economic subjects. These physical activities later initiate interaction within natural (material) outer environment and thus influence its self-organisation and evolution. Their meaning is derived from (limited) human knowledge and their impact is growing together with increasing amount of used energy. On the other hand, conceptual information actively takes part in forming the social reality, human cognition and knowledge (knowledge sets), as stated by Berger and Luckmanen [3]. Let's have a look at an example: In democratic societies, knowledge of individuals is projected to the elections' result, and this becomes reality for the next electoral term. This "reality" then influences not only social reality but also the real (physical) world. Within this context, the nature and meaning of knowledge and/or information plays vital role on self-organising of the environment (both socially and physically). In the process of self-organizing there is a factor of emergence of new, unexpected facts, which again have influence further development. Being aware of this (recursive) aspect is a necessity for better understanding and possible corrections.

\subsection{Recursive relation between information and knowledge}

In the field of information technology (or information systems) there is generally accepted the difference between data, information and knowledge. Their respective relation is demonstrated as a linear chain from data to knowledge (via information), sometimes there is Checkland and Holwell's [5] term "capta" (captured data) inserted as a second link in this linear chain.

$$
\text { ?? data } \rightarrow \text { (?) capta } \rightarrow \text { (?) information } \rightarrow \text { (?) knowledge ?? }
$$


The trouble is that this linear chain is oversimplified. It does not take into account questions related to the transformation of the respective phenomena (How do data become capta? What makes knowledge out of information (?), neither the meaning of the information.

The greatest fallacy, however, is that it makes one believe that there is causality involved, which is actually contrary to the reality. We would like to modify this scheme by introducing circular recursive relations between information and knowledge reflecting individual nature of knowledge mentioned above.

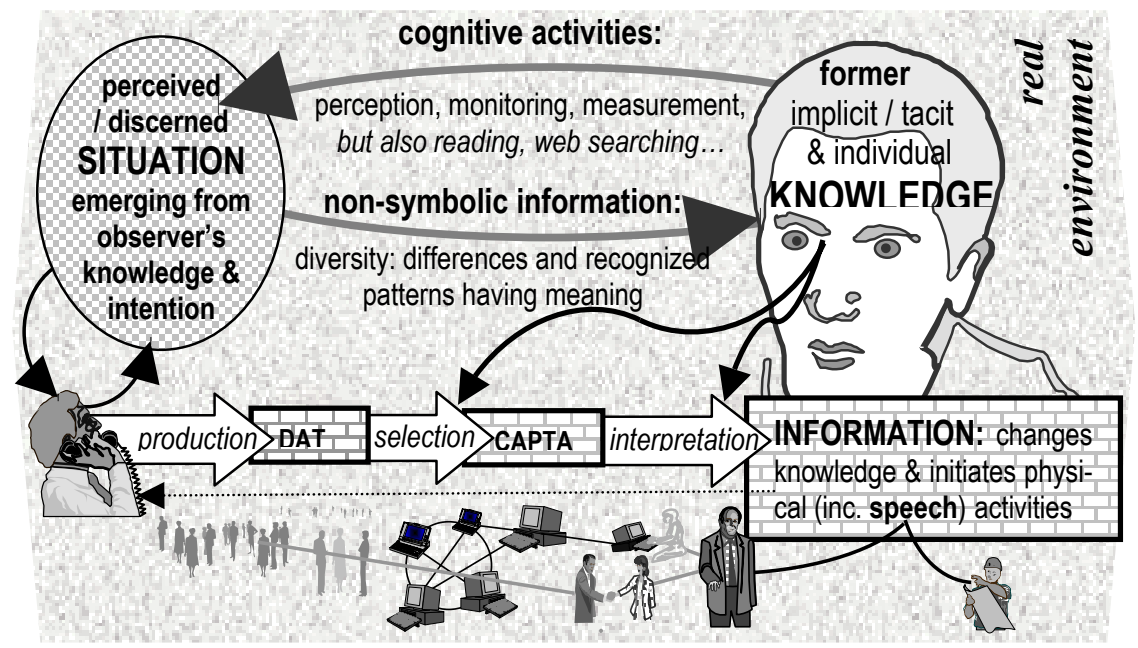

Figure 1: Circularity of information and knowledge.

Figure 1 puts the human into the position of the "observer", who actually lives in the global environment, where he can delimitate some situations based on his knowledge and intentions (Gestalt Psychology describes that as "delimitation"). This is fundamental principle of direct observation and knowledge-creation (represent by concepts and symbols). Symbols allow one to externalise knowledge: externalised knowledge can be shared through conceptual information; its meaning is demonstrated by the change of implicit knowledge and/or physical activities of the observer. Speech - a presentation of conceptual information in the form of language messages (data represents just the form constrained by syntactic rules) - is on the border-line of both. Here we come to the fact that any conceptual information is always presented by particular human - influenced by other humans, by his knowledge and by his intentions. Maturana and Varela [10, p. 27] expressively state: Everything said is said by someone.

So, once again, we stress the difference between data, symbols, and information: Information could have different meaning each and every time depending on the actual interpretation by the recipient. This process of interpretation could be influenced by the number of aspects - for instance by non-symbolic information, unconsciously perceived by the observer. 
The meaning of information is not constant or fixed, it depends on (variable) knowledge of the recipient, on the context, and on the actual conditions, or situation. But these factors, since you can't predict them, neither properly describe them, seems to be often overlooked (especially by the techno-boosters). However, such wilful ignorance could have serve implications.

\subsection{Validity and credibility of information and knowledge}

The above mentioned problems are related to the questions of validity and credibility of information and knowledge. Since it is quite broad and complicated issue, in everyday life it seems to be reduced (oversimplified) to the question of truthfulness and information is valued just on the scale "true/false". However, on this limited scale it is sometimes almost impossible to distinguish disinformation, which seems to be true in almost all aspect, the only "false" thing is the intention of the author. Nonaka [13] points out to that by saying, that "Knowledge is justified true belief".

However, the term truth itself seems to be a bit tricky. There are four different philosophy theories/concepts, broadly accepted [20]:

Correspondence theory: Narrowly speaking, the correspondence theory of truth is the view that truth is correspondence to a fact. This concept of truthfulness is the most useful, when it comes to decision and activities. Decisions are always based on the previous knowledge (what already had happened), but these decisions shape the future. It's a question of the order and (system) development, which gains the importance with the growing complexity of global world.

Coherence theory: A coherence theory of truth states that the truth of any (true) proposition consists in its coherence with some specified set of propositions. The coherence theory differs from its principal competitor, the correspondence theory of truth, in two essential respects. The competing theories give conflicting accounts of the relation between propositions and their truth conditions.

This theory is "in coherence" with the basic principles of (binary) computing and IT/ICT in general. Binary code does distinguish just two possible states ( 0 and 1$)$ and in accordance with it is the design of logical circuits. The computing is based on series of principles rules and possible operations, with determined results.

Consensus theory: Consensus theory of truth states that a statement is true if it would be agreed to by those who investigate it. So, it is just a matter of agreement in some social group (or culture). This theory reminds us of the hopes that designers and programmers put into the groupware as a technological tool to support group knowledge or group decision. But it actually does not answer how the consensus should be achieved (principles of democracy). It usually is taken for granted, that majority $(>51 \%)$ sets the course.

Pragmatic theory: The pragmatic theory of truth states that a belief or idea is true solely based on whether it works. This utilitarian, strictly anthropic and egocentric approach actually misses the global (including development in time) implications and context. However, pragmatic theory seems to be respected in business (entrepreneur) sphere, thanks to the relative importance of money (i.e. symbols). In IT/ICT sphere we can sense this approach in the attempts to 
"measure" benefits of IT/ICT, regardless to the actual meaning of information and knowledge.

None of the above theories explains a universal (absolute) concept of truth, which just demonstrates that there is no such thing as the only possible absolute truth, however confusing, uneasy (or even unacceptable) it could for some people be.

The reality is that human cognition and knowledge, or more precisely the conceptual information derived from them, is always limited and tied to the values and intention of the human. This is routinely accepted in modern systemic science and cybernetics, as well as in the knowledge management. There are three major reasons why:

- Limited (physically) abilities of human senses (receptors) - even when sometime artificially enhanced (telescope, listening devices, etc.)

- Intentional essence of the knowledge - and thus creating/constitution of social knowledge patterns.

- Evolutionary character of the world (universe) causes, that the growing complexity brings along the development, which does not match previous (earlier formulated) situation.

These facts are in contrary with the traditional idea, that information reduces uncertainty. The traditional concept of information (including Shannon's theory) has lost its importance and influence. Its supporters actually tend to argue, that better informed-ness could be achieved by massive using of IT/ICT.

The thing is that usage of IT/ICT brings us the bigger volume of data, however data are not knowledge. This over-saturation by data does not increase our knowledge: on the contrary, it actually reduces it. There is problem of relevance, validity and credibility. Moreover, the recipients usually lack the appropriate knowledge to interpret data correctly, so they are actually misinterpreted. So, the increased complexity of the modern, global world is affecting our society.

\section{Information, systems and evolutions}

These three terms (information, systems, evolutions) are used as the title for the last part of our paper on purpose. Evolution represents natural development, which originates in continuous and never-ending interaction of system (autonomous units) and their components. In these processes, information (including conceptual ones) plays a vital role, as they are interpreted by recipient, using his/her best individual knowledge (to achieve his/her intentions).

That is how human (social) systems differ from other systems. That is why human evolution is sometimes called "co-evolution". The result of human development is design of our artificial systems (including information systems, related to the IT/ICT). So, information systems are, and always have been considered as artefacts, as a thing you can easily handle. However, such understanding of an information system has recently been criticised. The answer lies in the concept of conceptual information. 
Technical (information) system - understood as a traditional artefact - has predefined all components and their possible properties. The organisation of the system is also carefully planed and prepared, so the expected inputs have the expected outputs, the behaviour of the system is perfectly predictable. Project (design) of the system comes from designer, whose knowledge is "embedded" into the project of system. When system is build, these ideas and knowledge is "embodied" in it. In case of IS/ICT system the embedded knowledge is represented by algorithms, database structure, software, etc.

Nevertheless, it's not only the technical (artificial) system that is designed and organised. Social systems, such as companies, businesses, schools, or press agencies are designed and planed as well. But, on the contrary, their functional elements are not things, but human beings, who produce, receive and interpret information according to their knowledge (thus individually). And they use ICT as well. Such systems are by Peter Checkland described as "systems of human activities" [4]. In these systems, information are NOT signals, but rather symbols. Participant's knowledge (not only professional, but all knowledge) are fundamental for the functionality of the system.

There are some specific "systems of human activities" whose main purpose is deal with information and/or knowledge: press agencies, media (editorial staff), schools (from elementary to university), research institutes, consultancy firms, etc. They are purposeful (designed, organised and directed) human information systems. Nevertheless, these information systems are not artefacts, but rather social institutions. And they process and mediate the information with some purpose, idea or reason "in mind". It would be foolish to expect, that they can provide some really "objective" or "neutral" information. It is simply beyond their reach, no matter how much they try. They can do their best to avoid some obvious bias, but since there will always be some sort of "embedded" knowledge, the output information must be biased somehow. And yet, outputs of these information systems are used by other people (general public) as inputs for their own decisions, for creating their own knowledge. The trouble is that there is not such thing as an assured quality of information. Neither can we independently access the information from all around the globe, no matter how many CNNs or BBCs we are watching. It is a wire service editor, who alone decides what news audiences will receive from another continent - and since there are just a few news or accidents, that actually have a "news value" high enough, to be broadcasted, it is probable, that these news will be accepted by almost all media. To put it as simple as possible: all information presented to us by media or any other human based information system, have been already "preselected", as described in agenda-setting and gatekeeping theory [12]. The idea is that when the gatekeeper's selections are biased, the recipients' understanding will therefore be a little biased as well. Nonetheless, there are some limits of how much can information be twisted (biased). It has to comply with the recipients' own knowledge and experience (weltanschauung), otherwise there is a danger, that it will be refused.

Advanced information technologies are especially vulnerable to be subject to the manipulation, since they are more convincing than the older ones. As an 
example could be mentioned a (falsified) video of hostage taking, that led to mass demonstrations of hundreds of thousands people.

Less evident (and perhaps even not purposeful) is the case of published photography of Palestinian boy being arrested by Israeli troops. The boy is crying, scared and it is clearly visible that he had wet his trousers. It's a very emotional picture that has flown round the globe and surely influenced the public opinion on Gaza Strip. The picture is surely true - so the impression must also be truthful, one might say. How sad it is that another picture, taken just a few moments before, received no attention. The very same boy, with the mask covering his face, is attacking the troops. It seems that there is more than one truth.

Let's just remind that the interpretation of provided information (data) is very individual activity of the receivers. That actually means that the very same data (information) will be interpreted in very different ways by different recipients. That (in our previous terminology) opens the possibility of conflicts and fluctuation within the system. The bigger are differences in interpretation (e.g. on the border between different cultures), the bigger are potential conflicts. This topical and up-to-date issue is very sensitive and should warn us to underestimate the problems of different understanding (or better say misunderstanding) in the globalized world. It makes us re-thing all series of issues, since similar problems are affecting everyday life of individuals, companies and society.

What we can see now, is that our social system is getting better organised. Thanks to the knowledge, embedded into different information systems (norms, standards, IS/ICT). That reduces the spontaneity and the ability to react (adapt) to the unexpected problems. This is the result of formalizing knowledge, which later (once saved into the system) does not change accordingly to the changes (development) of the system. The only way, how to keep necessary flexibility, is in human cognition (knowledge), which will be later used, when the information will be retrieved from the system and applied.

A similar sentiment, but in different terms, was expressed by Giddens [6], when he points out that our modern live is much more dependent on shared expertise and instructions that no longer match the complexity of contemporary world. His idea was further developed by Beck [2], who demonstrates that in modern society there are usually plenty of different opinions but the problem is to choose the right one. That is what Beck calls Risk Society.

\section{Conclusion}

Globalisation is closely connected to the complexity, thanks to the ease of sharing data globally via modern technology. But it raises the fluctuations in the system, since we loose our "natural" ability to react accordingly to our "shared" knowledge. We just rely on the (correct) information, knowledge and universal solutions, regardless of variability of reality. We loose our ability to react in a creative way. The Internet is a very typical phenomenon of this deterioration. The Internet is non-designed, hardly controllable information system that gives people great possibilities, but at the same time presents a great danger. 
The biggest problem is its anonymity, since it is very hard to actually verify the source of information.

The only way f how to deal with the threads and opportunities it gives us is concentrate on the nature of human knowledge. Current paradigms seems to fail when face the increasing complexity of modern world. As Peter Senge [17] describes:

"...blind technological progress exacerbates these problems, because it contributes more complexity when we cannot understand the complexity that already exists". We are out of control, driving down a dark road with little or no light, and most technological progress amounts to speeding up."

But we shall not blame information and communication technology. Instead we should apply "new conceptual understanding of complex systems". A suitable solution lies in the knowledge, and in the understanding properly to the actual meaning of the mediated information. Our short paper supported by Czech Science Foundation (project No 409025) hopefully offers an impulse to think about it.

\section{References}

[1] Bateson, G. Steps to ecology of Mind, Northvale, Jason Aronson, 1972.

[2] Beck, U. Risk Society - Towards a New Modernity, Sage Publication, 1992.

[3] Berger, P. \& Luckman, T. The Social Construction of Reality. A Treatise in the Sociology of Knowledge, Doubleday, New York, 1966.

[4] Checkland, P., Scholes Soft Systems Methodology in Action, J. Wiley, Chichester, London, 1990

[5] Checkland P., Holwell, S. Information, Systems and Information Systems, Wiley, Chichester, 1998.

[6] Giddens, A. The consequences of modernity, Polity Press, Cambridge, 1990

[7] Hayek, F.A. Law, Legislation and Liberty, Routletge, London, 1970.

[8] Hofkirchner, W. (ed.) The Quest for a Unified Theory of Information, Gordon \& Brecach, 1996.

[9] Krogh von, G. Ichijo, K. Nonaka, I. Enabling Knowledge Creation, Oxford Univ. Press, London, 1996.

[10] Maturana, H., Varela, F. The Tree of Knowledge, Shamhala, Boston, 1998.

[11] McLuhan Understanding Media, Routletge and Kegan Paul, 1964.

[12] McCombs, Maxwell E. and Donald L. Shaw Structuring the unseen environment, Journal of Communication, v. 26 no. 2, pp. 18-22, 1976.

[13] Nonaka, I. A Dynamic Theory of Organizational Knowledge Creation, In: California Management Review, 5 (1) 14-37, 1995.

[14] Paul, R., Doder, L. Critical Thinking, Prentice Hall, London, 2001.

[15] Rosický, A. Information generating system: Towards concept of information generating social systems, EMCSR, Vienna, 2002.

[16] Searle J. Mind, Brains and Science, Harvard University Press, 1986. 
[17] Senge, P., Through the Eye of the Needle, in: Gibson, R. (ed.) Rethinking the Future, Nikolas Breadley Pub., London. 1996.

[18] Shannon, C., Weaver, W The Mathematical Theory of Communication, University of Illinois Press, Urbana, USA, 1949.

[19] Stonier, T. Information and the Internal Structure of the Universe, Springer, UK 1990.

[20] Stanford Encyclopedia of Philosophy http://plato.stanford.edu.

[21] Wiener, N. Cybernetics or Control and Communication in the Animal and the Machine, MIT Press, Massachusetts, 1948.

[22] Winograd, T. and Flores, F. Understanding Computers and Cognition, Ablex Pub. Norwood, 1986. 